\title{
Mediävistische Lingolitologie am Beispiel fremdsprachiger Ortsnamen bei Wolfram von Eschenbach
}

\author{
Nathanael Busch
}

Angenommen: 7. Januar 2021 / Online publiziert: 26. Januar 2021

(C) Der/die Autor(en) 2021

Zusammenfassung Wolfram von Eschenbach greift für die Namenbildung auf verschiedene Quellen zurück und passt sie an das deutsche Phoneminventar an. Anhand dieses Beispiels wird nach den Bedingungen von Schnittstellen zwischen Literaturwissenschaft und Linguistik gefragt. Obschon die Mediävistik sich stets mit Sprache und Literatur zugleich auseinandersetzt, bleibt die Beschäftigung meist institutionell, personell und thematisch vereinzelt. Für eine gegenseitige Wahrnehmung wäre es hilfreich, bei der Formulierung von Ergebnissen und Erkenntnisinteressen ein breiteres Fachpublikum im Auge zu behalten.

Schlüsselwörter Germanistik · Mediävistik · Onomastik · Wolfram von Eschenbach: Parzival · Wolfram von Eschenbach: Willehalm

\section{Lingolitology in Medieval Studies Exemplified by Foreign Language Place Names in the Works of Wolfram von Eschenbach}

\begin{abstract}
Wolfram von Eschenbach makes recourse to proper name constructions out of various sources and adapts them to the inventory of German phonemes. These examples elicit questions as to the circumstances surrounding the intersection of literary studies and linguistics. Even though both language and literature are constantly grappled with in medieval studies, this dual engagement has remained institutionally, personally and thematically isolated. Keeping in mind a broader audience, consisting of representatives of diverse disciplines, while formulating conclusions and interests of academic insights would promote heightened mutual awareness.
\end{abstract}

Keywords German Studies · Medieval Studies · Onomastics · Wolfram von Eschenbach: Parzival · Wolfram von Eschenbach: Willehalm

N. Busch $(\bowtie)$

Institut für deutsche Philologie des Mittelalters, Universität Marburg, Marburg, Deutschland

E-Mail: buschn@uni-marburg.de 


\section{Mediävistik = Sprache und Literatur des Mittelalters?}

Erst auf den zweiten Blick erscheint mir als Mediävist die Vorstellung, die Beschäftigung mit Sprache und Literatur könnten streng voneinander geschieden sein, nicht mehr irrsinnig. Mein erster Blick ist noch unverstellt: Die Beschäftigung mit mittelalterlicher Sprache gehört zum Initiationsritus angehender Germanistinnen und Germanisten; erst im Anschluss darf man zur eigentlichen Lektüre vordringen. Auch wenn sich die Gegenstände der sogenannten Einführung, und mit ihnen das Fach, in den letzten Jahrzehnten enorm gewandelt haben und für historische Worterläuterungen (von >Alternanz /e/ - / i/ < bis >Westgermanischer Gemination $<$ ) kein Platz mehr ist, so bleibt die äußere Wahrnehmung des Teilfachs von der Sprache geprägt. Eine nicht geringe Zahl auch fortgeschrittener Studierender bedient sich der Formulierung, ein Seminar in >Mittelhochdeutsch $<$ zu belegen, nicht etwa ein mediävistisches.

Im alltäglichen Umgang mit mittelalterlichen und frühneuzeitlichen Texten kommt man nicht umhin, sich intensiv mit sprachlichen Phänomenen auseinanderzusetzen. Ein Beispiel aus der Praxis ist schnell gefunden. Anlässlich einer Anthologie zum Thema >Geld in der Literatur des Mittelalters< übersetze ich Hans Folz' Jüdischer Wucher. In diesem Text ergeht sich der Nürnberger Dichterbarbier in den widerwärtigsten Stereotypen, unter anderem spricht er davon, dass ein zurückbezahlter Kredit sogleich einem anderen Schuldner aufgenötigt werde. Entgegen anderslautender Gerüchte ist das Frühneuhochdeutsche tückisch, denn es ist ausdrucksreich, und die vorgefundenen Graphien sind nicht immer leicht einem bekannten Wort zuzuordnen:

Da merck: so dick das [= den Kredit] einr ablöst,

Wirt es eim andern zugenöst.

Und ob es von der keim geschicht,

Feiert der jüd doch trunter nicht. ${ }^{1}$

Scheinbar ist jedes Wort verständlich, tatsächlich jedoch ist der Zusammenhang schwer zu erfassen. Die Ursache der Schwierigkeit liegt im Wort keim, das nichts mit Setzlingen oder dem Indefinitpronomen >kein essich $\ll^{2}$ zu tun hat, sondern eine abfällige Bezeichnung für Juden aufruft, die sich vom Personennamen >Chaim< ableitet. ${ }^{3}$ Um solche Stellen zu entschlüsseln, benötigt man eine genaue Kenntnis von Spezialwörterbüchern wie auch der Grammatiken, aber auch ein profundes kultur- und literaturgeschichtliches Wissen. Wer mit Quellen arbeiten will und sie nicht als Anlass für von ihnen losgelöste Gedanken nimmt, kommt nicht umhin, sich mit Sprache und Literatur zu beschäftigen.

\footnotetext{
1 Folz, Hans: »Jüdischer Wucher«. In: Ders.: Die Reimpaarsprüche. Hg. von Hanns Fischer. München 1961, S. 310-318, hier: Verse 149-152.

2 Grimm, Jacob und Wilhelm: Deutsches Wörterbuch. 16 Bde. Leipzig 1854-1960, Bd. 11, Sp. 451, s.v. $>$ keim<.

3 Vgl. Klepsch, Alfred: Westjiddisches Wörterbuch. 2 Bde. Tübingen 2004, S. 775. Althaus, Hans Peter: Kleines Lexikon deutscher Wörter jiddischer Herkunft. München 2003, S. 57.
} 
Man könnte folglich meinen, in der Mediävistik sei das Paradies einer fruchtbaren Einheit von Linguistik und Literaturwissenschaft nie verlassen worden. Das Gegenteil ist der Fall, wie ein zweiter Blick verrät, der auf einen jener Publikationsorte fällt, an dem eigentlich beide Teilfächer vereint sein sollten. In den Beiträgen zur Geschichte der deutschen Sprache und Literatur versammelt sich dem Zeitschriftentitel zufolge die diachrone bzw. geschichtliche Forschung beider Richtungen. Doch die Lektüre der Inhaltsverzeichnisse der letzten Jahrgänge wirkt ernüchternd. Die Aufsätze lassen sich nur schon dem Titel nach in eine linguistische oder eine literaturwissenschaftliche Schublade sortieren; ein Blick auf die Autoren und Institutionen bestätigt die säuberliche Trennung der Ansätze. Es soll nicht unterstellt werden, dass die Verfasser die Chance einer Schnittstelle nicht ergreifen wollten; sie hatten sie einfach nicht im Blick. Kurioserweise unterscheidet selbst die Zeitschrift für Literaturwissenschaft und Linguistik im Merkblatt »zur Gestaltung der Beiträge « zwischen Literaturangaben in linguistischen und literaturwissenschaftlichen Beiträgen, die sich im Wesentlichen dadurch unterscheiden, ob die Jahreszahl vorne oder hinten steht. Dieselbe Trennlinie zeigt sich im vom DAAD geförderten Germanistenverzeichnis. ${ }^{4}$ Viele Mediävistinnen und Mediävisten geben zwar das Spezialgebiet >ältere Sprachstufeく an, >Sprachgeschichteく dagegen wählen sie nicht aus.

Diese institutionelle Grenze findet sich schließlich auch bei den beiden größten Prestigeprojekten mediävistischer (Sprach-)Forschung der letzten Jahre wieder. Das Mittelhochdeutsche Wörterbuch entsteht in zwei Arbeitsstellen in Göttingen und Trier, finanziert durch das Akademienprogramm. Das Vorhaben geht in die 1990er Jahre zurück und hat gerade den zweiten von fünf geplanten Bänden abgeschlossen. Die Mittelhochdeutsche Grammatik geht in dieselbe Zeit zurück, wurde als Langzeitvorhaben von der DFG gefördert und hat ebenfalls zwei Bände vorgelegt. Beide Publikationen stellen dringende Desiderate der Mediävistik dar, weil die wichtigsten Hilfsmittel unserer Zunft hoffnungslos veraltet sind. Sie sind nicht nur alt - die Wörterbücher gehören zur Gründungsgeschichte der Germanistik als Wissenschaft -, sondern beruhen auf überholten Quellen und Methoden. Der Nutzen beider Großprojekte ist evident, und die gigantischen, jahrzehntelangen Anstrengungen werden sich auszahlen, insofern das Mittelhochdeutsche nach Abschluss der Arbeiten nicht mehr dasselbe sein wird wie zuvor. Man müsste freilich das Wort >Mittelhochdeutsch $<$ in den Plural zu setzen, denn die Annahmen beider Vorhaben passen nicht recht zusammen. Das beginnt mit der augenscheinlichen Oberflächlichkeit, dass Längenzeichen in den Lemmata des Wörterbuches nach mediävistischem Usus mit Zirkumflex (̂) bezeichnet werden, in der Grammatik dagegen mit dem in der Linguistik eingeführten Makron (5). Weit gravierender aber ist die Wahl der zugrundeliegenden Quellen wie auch der Umgang mit ihnen. Das Wörterbuch arbeitet mit vorliegenden Editionen, zieht also einen nach philologischen Maßstäben hergestellten Text heran und bietet eine »möglichst umfassende [...] Beschreibung des mittelhochdeutschen Wortschatzes «. ${ }^{5}$ Die Grammatik dagegen beruht auf einem Korpus repräsentativer

\footnotetext{
4 http://www.germanistenverzeichnis.phil.uni-erlangen.de/ (03.09.2020).

5 Gärtner, Kurt/Grubmüller, Klaus/Stackmann, Karl (Hg.): Mittelhochdeutsches Wörterbuch. 1. Band. Stuttgart 2013, S. VIII.
} 
Handschriften, die ohne Eingriff ausgewertet und beschrieben werden, wobei allerdings aufgrund der Quellenlage zentrale Werke der Literaturgeschichte konsequent ausgeklammert werden. Vor Jahrzehnten wurden Festlegungen getroffen, die für sich nachvollziehbar sind, die aber eine unterschiedliche Auffassung von Sprache zum Ausdruck bringen. Es unterscheiden sich nicht bloß die Quellen, sondern der Anspruch auf umfassende Beschreibung wie auch die Vorstellung von Darstellbarkeit von Sprache weichen voneinander ab. Kurz: Das Mittelhochdeutsche, das beide Vorhaben im Titel tragen, ist je ein verschiedenes.

Nun stellen an sich unterschiedliche Forschungsansätze einen Gewinn und Ausdruck einer lebendigen Wissenschaftskultur dar. Doch in der Konsequenz wird das Vorgehen die Benutzung der Werke erschweren. Es steht zu befürchten, dass die Grammatik primär von Linguistinnen und Linguisten, das Wörterbuch von Literaturwissenschaftlerinnen und Literaturwissenschaftlern verwendet werden wird. In dieser Hinsicht sind auch Zweifel am Aussagewert des oben genannten Beispiels aus der laufenden Folz-Edition angebracht. Handelt es sich bei der Frage nach dem Wort kaim überhaupt um eine linguistische Fragestellung oder wird nicht vielmehr die Sprachwissenschaft zur einfachen Auskunftsstelle degradiert, deren Zweck es folglich wäre, die erhabene Interpretation zu ermöglichen? In einer solchen Hierarchie begegnet man sich nicht auf Augenhöhe, wie der Philologe Joachim Heinzle bemerkt: »Für den Literarhistoriker ist die Sprachgeschichte, für den Sprachhistoriker die Literaturgeschichte eine $>$ Hilfswissenschaft $<. \ll^{6}$ Diese Ausgangslage ist bedrückend. Es sei daher im Folgenden anhand eines Beispiels nach den spezifisch mediävistischen Möglichkeiten der >Wahlverwandtschaft $<$ gefragt.

\section{Zum Beispiel fremdsprachige Ortsnamen bei Wolfram}

Die Eigennamen bei Wolfram von Eschenbach hinterlassen einen eigentümlichen Nachgeschmack. Wer den Parzival gelesen hat, wird sich an die verwirrenden Silbenketten erinnern, die Wolfram zu Namen zusammensetzte. Wo mag bloß Ipopotitikôn liegen? Wie mögen die Menschen in Gampfassasche aussehen? Wie soll man sich Namen merken können wie König Samirant von Boytendroyt?

Schon die schiere Quantität hat das Potential, einen in die Verzweiflung zu treiben. In seinem Gesamtwerk führt Wolfram 935 verschiedene Namen an, davon 515 Personen- und 420 Ortsnamen. ${ }^{7}$ In den Werkausgaben finden sich ausführliche Register, damit man den Überblick behält. Schon auf dem Buchdeckel der Paperbackausgabe des Verlags De Gruyter schlüsselt ein Diagramm die komplizierten Verwandtschaftsverhältnisse auf. Die Namen spannen ein ganzes Wissensnetz durch seine Texte, das in seiner Komplexität bei Chrétien nicht vorgezeichnet ist; der Handlungsraum ist

\footnotetext{
${ }^{6}$ Heinzle, Joachim: »Über die wechselseitigen Beziehungen zwischen Sprachgeschichte und Literaturgeschichte«. In: Jahrbuch für germanistische Sprachgeschichte 1 (2010), S. 188-204, hier S. 190.

7 Einen Überblick zu den arthurischen Werken bietet Schröder, Werner: Die Namen im >Parzival< und im >Titurel Wolframs von Eschenbach. Berlin [u.a.] 1982. Im Folgenden zitierte Ausgaben: Wolfram von Eschenbach: Parzival. Hg. von Karl Lachmann. Berlin ${ }^{6} 1926$. Wolfram von Eschenbach: Willehalm. Hg. von Joachim Heinzle. Frankfurt a.M. 2009 [Namenverzeichnis zum Willehalm im Anhang].
} 
laut dem Mediävisten Joachim Bumke durch eine Fülle realer Orts- und Ländernamen so strukturiert, dass er die ganze damals bekannte Welt umfasse. ${ }^{8}$ Dennoch, so mag man ergänzen, weiß man nie, ob der Erzähler sich gerade einen Jux daraus macht.

Wolfram pflegt einen spezifischen, beschreibbaren Umgang mit Namen, der für seine Romane charakteristisch ist. Man möchte folglich wissen, was das Charakteristikum dieses spannenden Wortmaterials ausmacht. Ehe man sich einer Untersuchung zuwendet, muss zuvor dem Umstand Rechnung getragen werden, dass es sich um Namen in Literatur handelt - und eben nicht um Material aus Urkunden oder Chroniken. Es gilt zunächst, den Status des Wortmaterials zu bestimmen, denn Namen sind nicht Wörter wie andere Wörter. Für die Linguistin Damaris Nübling stellen sie eine sprachliche Sonderkategorie dar, die beispielsweise über eine eigene Grammatik und eine spezielle Form der Referenzierung verfügt. ${ }^{9}$ »Nomen est omen «, weiß das Sprichwort. Mit dem Namen fängt alles an, manche sagen sogar, das Leben selbst beginne mit dem Namen. ${ }^{10}$ Obgleich doch die Benennung, so könnte man meinen, ein außersprachliches Objekt mit einem zufälligen Zeichen in Verbindung bringt, scheint der Namengebung und der Namennennung noch immer ein Zauber innezuwohnen, der das Leben festlegt. Mit dem Namen erschließt sich einem, so diese Vorstellung, zugleich das Wesen der Dinge. Nomina sunt consequentia rerum, heißt es bei Dante. ${ }^{11}$

Jenseits dieses Aberglaubens kann die heutige Gesellschaftswissenschaft nachweisen, wie Namen mit sozialen Praktiken verbunden sind. In einer Zeitungsnotiz vom August 2010 ist zu lesen, dass Pädagogen der Universität Oldenburg eine Korrelation zwischen Vornamen und schulischer Notengebung ermitteln konnten. »Kevin« und »Celina« hätten dabei schlechter abgeschnitten als »Maximilian« und »Charlotte «. ${ }^{12}$ Dieser Befund wird wohl kaum so gedeutet werden können, dass diese Namenträger per se, also ihrem Wesen nach, schlechtere Schüler seien, sondern dass die Namen mit sozialen Konstellationen einhergehen oder darin scheinbar wiedererkannt werden. Durch das Anrufen mit diesen Namen wird das Individuum also nicht bloß ein Subjekt, sondern bereits schon ein konkretes, typisches Subjekt.

Schriftsteller können sich dieses Verhältnis zum Namen bewusst zu eigen machen. Sie können Namen zum Sprechen bringen (z.B. in reinen Klangphänomenen wie Kuddeldaddeldu, in semantischer Ausdeutung wie bei Gottfried von Straßburg: von triste Tristan was sîn nam ${ }^{13}$ oder in einer Rezeptionslenkung, wie sie bereits in einem

\footnotetext{
8 Bumke, Joachim: Wolfram von Eschenbach. Stuttgart/Weimar ${ }^{8} 2004$, S. 201.

9 Vgl. Teil I des Bandes Nübling, Damaris/Fahlbusch, Fabian/Heuser, Rita: Namen. Eine Einführung in die Onomastik. Tübingen ${ }^{2} 2015$.

10 Einige Zitate zum Namen »als konstitutives Element der Lebenswelt « finden sich bei Debus, Friedhelm: Namen in literarischen Werken. (Er-)Findung - Form - Funktion. Stuttgart 2002, S. 9-11.

11 Dante (Vita Nova XIII,4) bezieht sich auf Justinian (Instutio 2,7,3), zum philosophiegeschichtlichen Hintergrund vgl. Flasch, Kurt: »Die Pest, die Philosophie, die Poesie«. In: Walter Haug/Burghart Wachinger (Hg.): Literatur, Artes und Philosophie. Berlin/Boston 1992, S. 63-84, hier S. 78.

12 N.N.: »Schlechtere Noten für Kevin«. Süddeutsche Zeitung vom 24.08.2010. In: http://www.sueddeut sche.de/karriere/vorurteile-gegen-vornamen-schlechtere-noten-fuer-kevin-1.991866 (30.08.2020).

13 Gottfried von Straßburg: Tristan und Isolde. Hg. v. Friedrich Ranke. 1. Bd.: Text. Berlin 1930, Vers 2003.
} 
Titel aufgerufen wird wie Wilhelm Meisters Lehrjahre). Vor allem können sie mit den Namen typische Figuren auftreten lassen, deren Konnotat nicht bloß auf persönlichen Erfahrungen beruht. »Klassifizierende Namen « nennt Hendrik Birus solche Namen, ${ }^{14}$ ihre Träger werden aufgrund bestimmter Namensgebungskonventionen einer Gruppe zugeordnet.

Es sei, so Birus, in Gryphius' Komödien auch ohne Kenntnis der näheren Personenkennzeichnung offensichtlich, dass Isaschar einen Juden bezeichne. Das gilt noch mehr für Ortsnamen, denn sie sind nicht beliebig ersetzbar. Wie der Kieler Linguist Friedhelm Debus anmerkt: Ein Name muss passen, er muss stimmig sein. »Eine in Süddeutschland angesiedelte Handlung kann nicht in Orten lokalisiert werden, die z.B. auf -büttel oder -leben enden, wie umgekehrt eine norddeutsch-niederdeutsche Geschichte in einem Ort auf -kofen deplaziert wäre. ${ }^{15}$ Mit Orten sind dann bestimmte Geschichten verbunden. Man spiele nur ein wenig mit Buchtiteln und stelle fest, dass eben der Mord im Orientexpress etwas Spannenderes verheißt als ein Mord im Main-Lahn-Sieg-Express. Und Thomas Mann hat, Gottseidank, nicht den Tod in Eckernförde geschrieben. -

Geht die Namenkunde traditionell von der Sprachwissenschaft aus, bietet die Untersuchung von Namen in literarischen Texten, die literarische Onomastik also, ein Forschungsgebiet zwischen Literatur- und Sprachwissenschaft, das einerseits die genaue Wortform (historisch) zu erläutern hat, zugleich aber stets im Hinterkopf behalten muss, dass das untersuchte Material literarischen Texten entstammt, die in ihrer Eigengesetzlichkeit ernst genommen werden müssen. ${ }^{16}$ Man darf also nicht bloß nach dem >Umgang < Wolframs mit fremdsprachigen Ortsnamen fragen, sondern hat neben diesem Erkenntnisziel gleichberechtigt ein weiteres Interesse zu stellen, nämlich die Frage nach der Funktion dieser Namen im literarischen Text. Wie Wolfram Raum entfaltet, wurde in den letzten Jahren verstärkt analysiert, und man kann auf die Ergebnisse zurückgreifen. Dazu noch einmal Bumke: Der Raum sei im Parzival ein Mittel, mit dem Zusammenhänge und Sinn erkennbar gemacht werden. ${ }^{17}$ Ein Mittel, mit dem dieser Zusammenhang hergestellt werden kann, sind Ortsnamen.

Wolframs fremdsprachige Toponyme können grob in zwei Bereiche unterteilt werden: Zunächst sind da die französischen Ortsnamen. Dieser Bereich liegt nahe, da die Vorlagen des Parzival und des Willehalm, also Chrétiens Perceval sowie die anonyme Chanson de Geste Aliscans, in Altfranzösisch verfasst wurden. Der zweite Bereich enthält arabische Namen, die prominent bei der Orientfahrt Gahmurets im Parzival sowie generell bei der Auseinandersetzung mit den Heiden im Willehalm vorliegen.

\footnotetext{
14 Birus, Hendrik: Poetische Namengebung. Zur Bedeutung der Namen in Lessings >Nathan der Weise Göttingen 1978, S. 37.

15 Debus: Namen (s. Anm. 10), S. 64-66.

16 Neu dazu Kohlheim, Volker: Der Name in der Literatur. Unter Mitarbeit von Rosa Kohlheim. Heidelberg 2019.

17 Bumke: Wolfram (s. Anm. 8), S. 201.
} 
Zunächst die französischen Ortsnamen, ${ }^{18}$ die sich in zwei Typen unterteilen: einerseits die von der Vorlage übernommenen und andererseits die von Wolfram selbst eingesetzten Namen. Die Wörter der zweiten Typs erlauben Erkenntnisse über Wortbildung von Fremdworten, dagegen verweisen die von anderen Quellen übernommenen Namen sowohl auf die linguistischen Aneignungsprozesse einer Fremdsprache (Stichwort: Sprachkontakt) wie auch auf deren Vermittlungswege (Stichwort: Kulturkontakt).

Im Hintergrund lauert jene Diskussion, die in der Forschung teils heftig geführt wurde, um Wolframs Bildung. Die zahlreichen Voten lassen sich zwischen zwei Extrempositionen anordnen: Entweder war Wolfram Analphabet oder er war gebildeter Litteratus. Für die Untersuchung hier spielt eine Festlegung zwischen den beiden Polen weniger eine Rolle als die Beachtung der Konsequenzen dieser Problematik. Gerade für die Graphie von Eigennamen ist es entscheidend, ob er über eine schriftliche Vorlage verfügte oder ob ihm die Geschichte mündlich erzählt wurde. Bei einer mündlichen Übertragung wären Hör- und Erinnerungsfehler vorprogrammiert, die sich in der unmotivierten Veränderung des Lautmaterials sehen ließen. Dazu ein Beispiel. Gawan muss an einer Stelle eine Furt überwinden, obgleich man sie im Sinne der Erzähllogik gar nicht durchqueren kann. Gawans Lösung: Er springt einfach über die Furt. Chrétien nennt diese Stelle mit einem Appellativum Li Guez Perilleus (»die gefährliche Furt«). Wolfram verwandelt diese französische Beschreibung in den französischen Namen der furt Ligweiz Prelljus. ${ }^{19}$ Was lässt die Lautgestalt erkennen? Gegenüber Chrétiens Form li Guez Perilleus haben folgende Änderungen stattgefunden:

1. Li. Der Artikel ist Teil des Namens geworden. Sollte das tatsächlich das Produkt eines ignoranten Stümpers sein, der nicht wusste, wie französische Artikel aussehen? Eine solche These wäre indes kaum belastbar: Es ist nicht vorstellbar, dass ein Übersetzer oder Vorleser Wolfram die Geschichte vermittelte und dabei nicht die Bedeutung dieser Worte erläuterte.

2. guez $>$ gweiz. Möglicherweise verwechselte Wolfram den Laut mit jenem charakteristischen französischen Diphthong -eis/-ois wie in rois oder kurtois, ${ }^{20}$ denn dieser wird im Deutschen oft mit der älteren Form ei wiedergegeben (reis, kurteis). Man kann dem Wort also die Prinzipien einer bestimmten Wortbildung ablesen, die kurzerhand auf ähnliche, aber anders gebildete Wörter übertragen wurde.

3. er $>$ re (in Perilleus). Es handelt sich bei der Umstellung um eine Metathese, die sich oft in höfischer Sprache findet, beispielsweise in ors und $\operatorname{ros}^{21}$ Die Metathese wird vom Niederländischen bzw. Niederdeutschen in den Süden übernommen: Könnte es sich um einen Hinweis auf eine pikardische Vorlage handeln?

\footnotetext{
18 Grundlegend: Knapp, Fritz Peter: »3. Graphematik - Phonologie«. In: Réne Pérennec (Hg.): Germania Litteraria Mediaevalis Francigena. Band 2: Sprache und Verskunst. Berlin/Boston 2014, S. 87-106 sowie Pérennec, René: »5. Onomastik«. In: Ebd., S. 183-264.

19 Parzival 583,25.

20 Rheinfelder, Hans: Altfranzösische Grammatik. 1. Teil: Lautlehre. München ${ }^{5} 1976, \S \S 40-49$.

21 Paul, Hermann: Mittelhochdeutsche Grammatik, neu bearb. v. Thomas Klein/Hans Joachim Solms/ Klaus-Peter Wegera. Mit einer Syntax von Ingeborg Sch[r]öbler, neubearb. und erweitert v. Heinz-Peter Prell, Tübingen ${ }^{25} 2007$, §L91.
} 
4. eus $>$ jus. Der Diphthong wird im 13. Jh. im Französischen zu $\ddot{o}$ monophthongiert. ${ }^{22}$ Die hier übernommene Form zeigt sich von dieser Änderung noch nicht beeinflusst. Im Gegenteil, das $u$ wurde offenbar stärker betont als das $e$, das mit dem vorangehenden Laut verschmolzen ist.

5. Ferner ist zu beachten: $z / s$ und $l l$ ändern sich graphisch nicht. Sie haben aber im Deutschen nicht den gleichen Lautwert wie im Französischen: $l l$ ist dort ein palatales $t$ wie in Italienisch figlia. $z$ bezeichnet im Französischen in diesem Fall eine Affrikata, im Deutschen dagegen ist mit $s / z$ wahrscheinlich der Unterschied zwischen Lenis und Fortis desselben Reibelauts bezeichnet. ${ }^{23}$

Diese fünf Aspekte sind nicht einem Versehen oder einem Hörfehler geschuldet, sondern lassen Strategien erkennen. Wolfram hätte auch im Deutschen ein Appellativum bilden können: der schedelîch/übel vurt. Wolfram hat sich für einen anderen Weg entschieden. Offenbar war es wichtiger, den französischen Klang beizubehalten, oder genauer: innerhalb des deutschen Lautsystems den französischen Klang nachzuahmen. Sollte diese Vermutung zutreffen, kann man an den Wortformen tatsächlich Wortbildungsregeln erkennen. Es würde sich an diesem Material zeigen, wie der fremde Klang unter Berücksichtigung von Kenntnissen der französischen Sprache, die bei Autor und Publikum vermutet werden können, mit den möglichen Lauten und ihrer Verschriftung im Deutschen in Einklang gebracht werden.

Ein für moderne Ohren naheliegenderes Beispiel kann das verdeutlichen. Der Name Schampâne lässt sich problemlos dem franz. Champaigne zuordnen. ${ }^{24}$ Die Laute werden offenbar unter Berücksichtigung des lateinisch-romanischen PhonemInventars ans Deutsche angeglichen. Der charakteristische Diphthong [t $\mathrm{f}]$ im Anlaut wird zu einem einfachen Zischlaut [ $]$ ]. Das aus dem Diphthong stammende offene $[\varepsilon]$ (graphisch <ai>) entspricht im Deutschen einem Langvokal [a:]. Der neue, ebenfalls charakteristische präpalatale Nasal $[n]=<\mathrm{gn}>$ wird zurückgeführt auf einen einfachen Nasal, aus dem er ursprünglich im Lateinischen stammt.

Die Veränderungen und Anpassungen bei Ligweiz Prelljus und Schampâne erlauben allerdings keine Aussage über die Frage nach Wolframs Lesefähigkeiten. Man braucht die Änderungen nicht auf die Aussprache eines Vorlesers zurückzuführen, denn genauso ist denkbar, dass Wolfram sich die Vorlage selbst vorlas, sich dabei verlas oder mit den Klängen spielte. Dazu muss man sich in Erinnerung rufen, wie im Mittelalter gelesen wurde. Abschreiber wie Leser haben im Mittelalter die Texte zum Klingen gebracht, sie haben laut gelesen, ${ }^{25}$ d.h. sie mussten den Text nach dem ihnen gewohnten Lautsystem einer Sprache intonieren. Um diese Umsetzung besser zu verstehen, bräuchten wir umfassendere Kenntnisse sowohl über diesen Kopiervor-

\footnotetext{
22 Rheinfelder: Grammatik (s. Anm. 20), §55.

23 Rheinfelder: Grammatik (s. Anm. 20), §§53, 342, 679, 799. Paul: Grammatik (s. Anm. 21) §L120.

24 Den geläufigen Namen fand Wolfram vermutlich nicht in seiner Vorlage, denn er ist nur in epigonalen französischen Artusromanen belegt, vgl. West, G.D.: An Index of Proper Names in French Arthurian Prose Romances. Toronto 1978, S. 37.

25 Vgl. Balogh, Josef: »Voces paginarum. Beiträge zur Geschichte des lauten Lesens und Schreibens «. In: Philologus 82 (1927), S. 84-109, 202-240. Saenger, Paul: Space Between Words. The Origins of Silent Reading. Stanford 1997.
} 
gang wie auch insgesamt über französische Fremdwörter im Deutschen, die über die positivistischen Forschungen der ersten Hälfte des 20. Jahrhunderts hinausgehen. ${ }^{26}$

Doch das Wortmaterial erlaubt nicht nur Aussagen zu Wortbildungsregeln, sondern auch zu poetologischen Strategien. Das zeigt sich noch einmal mehr an den von ihm selbst erfundenen Namen, die er nicht von seiner Vorlage übernehmen konnte. Zum Beispiel Beafontane, die »schöne Quelle« also, formte er entsprechend den Bildungsregeln von Ortsnamen seiner Vorlage wie Beaterre (»schönes Land «, Willehalm) und Bearosche (»schöner Berg «, Parzival u.a.). ${ }^{27}$ Darüber hinaus erfand er auch Namen, die bloß französisch klingen sollen, die aber nichts bedeuten. Sie erlauben einen Einblick in jene Wortelemente, die als französisch galten. Ein Beispiel gibt die von Parzival am Ende seiner Geschichte aufgezählte Liste besiegter Gegner. Diese Liste umfasst beinahe einen ganzen Dreißiger und klingt von Vers zu Vers abenteuerlicher:

Von Lirivoyn den künec Schirnîel, und von Avendroyn sîn bruoder Mirabel, den künec Serabil von Rozokarz, und den künec Piblesûn von Lorneparz, von Sirnegunz den künec Senilgorz, und von Villegarunz Strangedorz, von Mirnetalle den grâven Rogedâl, und von Pleyedunze Landunâl, den künec Oniprîz von Itolac, und den künec Zyrolan von Semblidac $[. . .]^{28}$

Zunächst sieht man in diesem Auszug wiederum, wie mit den Wortendungen eine frankophone Etymologie evoziert wird: -oyn/-ôn, -arz/-orz, -unz(e), -alle, -ac, -îs. Wolfram verpasst den erfundenen Namen ein französisches Gepräge, doch die Wörter sind nun vollends beliebig. Es handelt sich um Namen, die auf nichts mehr verweisen außer auf sich selbst, sie erzeugen nichts als einen Klangteppich oder ein vormodernes Grammelot im Stil von Dario Fo. Vereinzelt ist versucht worden, in den Wörtern eine Bedeutung zu finden. ${ }^{29}$ Mit Sicherheit ist hier aber nicht von einer tatsächlichen Lokalisierung des Geschehens auszugehen, sondern Wolfram greift auf eine ältere literarische Praxis zurück: diejenige der Namenlisten und Heldenkataloge, wie sie sich schon in der antiken Epik finden und zu deren bekanntesten Vertretern die Schiffskataloge der Ilias oder die Heldentatenlisten in der Aeneis zäh-

\footnotetext{
${ }^{26}$ Vgl. z.B. Öhmann, Emil: »Die französischen nomina propria in den deutschen Denkmälern des 12. und 13. Jahrhunderts«. In: Neuphilologische Mitteilungen 19 (1918), S. 9-16. Rosenqvist, Arvid: Der französische Einfluss auf die mittelhochdeutsche Sprache im 14. Jahrhundert. 2 Bde. Helsinki 1932/1943. Suolahti, Hugo: Der französische Einfluss auf die deutsche Sprache im 13. Jahrhundert. 2 Bde. Helsinki 1929/1933.

27 Beafontane: Parzival 125,16. Beaterre: Willehalm 359,1. Bearosche: Parzival u.a. 349,3.

28 Parzival 772,1-10.

${ }^{29}$ Beispielsweise Privegarz $=»$ beraube den Knecht $«$, Strangedorz $=»$ Schind' den Rücken $«$, vgl. Kleiber, Wolfgang: »Zur Namenforschung in Wolframs >Parzival««. In: Der Deutschunterricht 14.6 (1962), S. 80-90, hier S. 89 .
} 
len. Mit jedem Namen der hier nur zu einem Drittel zitierten Liste steigert sich der Ruhm Parzivals, auch wenn dem Vortrag ein gewisses komisches Potential nicht abgestritten werden kann.

Es ist gut möglich, dass Wolfram die Liste von einer bisher unbekannten Quelle übernahm. Denn das gilt für eine weitere Namenliste, die aus dem zweiten Sprachbereich stammt. Diese Liste findet sich nur zwei Dreißiger zuvor. In ihr zählt Parzivals Halbbruder Feirefiz die von ihm besiegten Gegner auf. Die Namen dort sollen folglich nicht mehr französisch klingen, sondern orientalisch bzw. arabisch - oder in Wolframs Sprache: heidenisch.

Der künec Papirîs von Trogodjente, und der grâve Behantîns von Kalomidente, der herzoge Farjelastis von Affricke, und der künec Liddamus von Agrippe, der künec Tridanz von Tinodonte, und der künec Amaspartîns von Schipelpjonte, der herzoge Lippidîns von Agremuntîn, und der künec Milôn von Nomadjentesîn, von Assigarzîonte der grâve Gabarîns $[\ldots]^{30}$

Die Liste orientiert sich wahrscheinlich an einer von Solinus im 3. Jahrhundert verfassten Mirabilien-Sammlung, die Namen sind folglich in der Hauptsache griechischer Provenienz. ${ }^{31}$ Bemerkenswert nun die Wortbildung: Die Wörter sind mit den entsprechenden Endungen versehen, damit sie zu einem Phantasieprodukt im Sinne eines klassifizierenden Namens werden können, wie Wolfram auch hier sämtliche Namen den lautlichen und graphematischen Gepflogenheiten des Deutschen anpasst. So wird beispielsweise aus den lateinischen Wörtern Nomades + gente + Adj. -inus der deutsche Name Nomadjentesîn. Aus Cyclopes + gentes wird Schipelpjonte, aus Cynodontas der Name Tinodonte. ${ }^{32} \mathrm{Zu}$ beobachten sind Endungen auf -în/-ôn/-ûn, d.h. jeweils ein Langvokal mit Nasal, oder auf -ente/-onte, d.h. Kürzung des Vokals bei Nasal + Plosiv.

Der fremde Klang der Wörter reichte offenbar aus, damit der gewünschte Effekt erzielt werden konnte. Es wäre dann gar nicht mehr relevant, ob die Namen tatsächlich griechischer oder orientalischer Natur wären, da die Griechischkenntnisse im deutschen Sprachgebiet ohnehin gering waren. Hinsichtlich tatsächlich arabischer Namen bietet sich einem beim späteren Wolfram ein anderes Bild. Sein zweiter großer Roman, der Willehalm, entstammt der Chanson-de-Geste-Tradition, in der der Kampf mit den Heiden ein tragendes Element bildet. Insofern ist es nicht verwunderlich, wenn in ihr mehr Orte gefunden werden, die dem arabischen Kulturraum zugeordnet werden können.

\footnotetext{
30 Parzival 770,1-9.

31 Wolfram von Eschenbach: Parzival. Nach der Ausgabe Karl Lachmanns, revidiert und kommentiert von Eberhard Nellmann. Übertragen von Dieter Kühn. 2 Bde. Frankfurt a.M. 1994, S. 764 (Stellenkommentar).

32 Vgl. Schröder: Namen (s. Anm. 7), S. 87, 109, 118.
} 
Grundlegend anders verlaufen nun die Vermittlungswege. Denn Wolfram greift nicht mehr auf ein pseudoarabisches Griechischvokabular zurück. Exemplarisch soll das wiederum an einer Namenliste demonstriert werden.

der künec Mattabel von Tafar.

der künec Kastablê von Komîs. [...]

der künec Tampastê von Tabrastên.

und der künec Goriax von Kordubin: [...]

der künec Haukauus von Nûbîâ [...].

Kursaus von Barberîe, [...]

der künec Bûver von Siglimessâ

und der künec Korsublê von Dannjatâ.

der künec Korsudê von Saigastin [...].

der künec Urabel von Korâsen [...];

der künec Hastê von Alligues [...].

der künec Embrons von Alimec;

künec Joswê von Alahôz. ${ }^{33}$

Wolfram übernahm diese Liste von seiner Vorlage, der Chanson de Geste Aliscans. Dort fand er allerdings nur die Personen-, nicht die Ortsnamen vor. Also fügte er letztere selber hinzu. Ein Beispiel: der künec Hastê von Alligues (74,21). In Aliscans findet sich bloß der Personennamen Astés (1178), ein Herkunftsname wird nicht angegeben. Abermals stellt sich die Frage, worauf sich Alligues beziehen könnte und woher Wolfram allenfalls den Namen übernommen haben wird. Wollten wir Germanisten die Wege tatsächlich orientalischer Wörter verstehen, wären wir hoffnungslos überfordert.

Es ist ein Glücksfall, dass der Orientalist Paul Kunitzsch in einer Reihe von Aufsätzen dieses Wissen entschlüsselt hat. Die Ergebnisse seiner Untersuchungen sind z.T. spektakulär. Die hier genannte Namenliste übernahm Wolfram mit großer Wahrscheinlichkeit en bloc der lateinischen Übersetzung eines Werks des arabischen Astronomen al-Fargān̄̄i. Kunitzsch konnte diesen Weg zurückgehen und dabei ermitteln, dass Alligues wohl al-Ahwâz entspricht, einer Landschaft mit gleichnamiger Hauptstadt im südwestlichen Iran. ${ }^{34}$

Die Entschlüsselung der Wortherkunft erhellt die Konzeption der Namenliste. Der literarisch konstruierte Raum unterscheidet sich deutlich von erfahrbaren Verhältnissen. Die aufgerufenen Orte sind breit über das gesamte arabische Gebiet verstreut, sie stehen in keiner Verbindung zueinander und können folglich nicht als Pendant eines realen Ortes interpretiert werden. Laut Hartmut Kugler, der sich mit geographischen Vorstellungen des Mittelalters beschäftigt, erlauben die punktförmigen geographischen Hinweise nicht die Konstruktion eines Flächenbildes - anders als im fiktiven

\footnotetext{
33 Willehalm 74,4-25.

34 Vgl. Kunitzsch, Paul: »Die orientalischen Ländernamen bei Wolfram (Wh. 74,3 ff.)«. In: Wolfram-Studien 2 (1974), S. 152-173, hier S. 165 (wieder in: Ders.: Reflexe des Orients im Namengut mittelalterlicher europäischer Literatur. Hildesheim 1996, S. 63-84).
} 
Gralsland, das mit Wolframs Angaben geradezu kartierbar wäre. ${ }^{35}$ Mit den orientalischen Toponymen werden dagegen keine geographischen Kenntnisse verbunden. Es muss um beliebige arabische Orte gegangen sein. Gleichwohl bestand ein Interesse, nicht mehr bloße Klangphänomene zu schaffen, sondern Wissensbestände zu übernehmen. Wolfram verleiht seinen Namen zuletzt eine besondere Authentizität.

Ich fasse die Ergebnisse des lingolitologischen Experiments zusammen: Wolfram entnimmt Namenmaterial nicht nur seiner direkten Vorlage, sondern greift auch auf weitere Quellen zurück. Geschickt passt er sie dem deutschen Phoneminventar an, bleibt dabei aber nicht stets der Vorlage treu, sondern spielt mit dem Vorgefundenen und ergänzt es. Wolfram setzte mit dieser Herangehensweise einen Standard. Nicht nur werden die Figuren seiner Texte von anderen Autoren aufgegriffen, auch in der Wortbildung macht er Schule. Der Wigamur-Dichter beispielsweise wirft etwa 50 Jahre später mit solcherlei Namen nur so um sich: Die juncvrouwe von Grabalmontois, künec Lipondrigûn von Gurgralet, Glakotelesfloir sowie Diatorforgranz. von Triasoltrifertrant sind nur einige von ihnen.

Das Studium des Namenmaterials nicht nur von Wolfram, sondern der mhd. Literatur überhaupt ist für die Mediävistik in seiner ganzen Breite attraktiv. Es verhilft zu einem Einblick in die Lebendigkeit und Beweglichkeit der höfischen Dichtersprache. Es bietet Möglichkeiten sowohl zu kulturgeschichtlichen Fragestellungen wie auch zu philologisch-sprachhistorischen Forschungen insbesondere in Hinblick auf Erkenntnisinteressen wie die Erforschung von Sprachkontakt und Mehrsprachigkeit, die weit über die oben angedeuteten lautgeschichtlichen Fragen hinausgehen. Die für die Mediävistik so grundlegende Perspektive der Materialität, die Überlieferungsforschung, muss dabei eine entscheidende Rolle einnehmen. Dieses Material müsste nur umfassend gehoben werden. Was für Wolfram weitgehend geschehen ist, müsste für weitere mhd. Werke nachgeholt werden. Ein ausführliches und zuverlässiges Namenverzeichnis ist trotz mehrerer Versuche bislang nicht zustande gekommen. Für die Forschung ist das ein Schaden. Dabei könnte es ein Projekt sein, das just jene Brücke schlägt zwischen Literaturwissenschaft und Linguistik. Es würde einen neuen Bereich linguistischen Materials umfassend erschließen und böte einen Überblick über die geographischen Wissensbestände in dieser Literatur.

\section{Schnittstellen}

Die Ortsnamen sind ein Beispiel für eine Schnittstelle zwischen Linguistik und Literaturwissenschaft. Es zeigt, unter welchen Bedingungen die Zusammenarbeit zwischen den Teilfächern möglich ist, und erlaubt Aussagen darüber, was von anderer Seite benötigt wird, damit man selber arbeiten kann. Um das Thema erst als solches zu erkennen und das Material weitergehend auszuwerten, wurde auf gängige Arbeitsmittel zurückgegriffen, beispielsweise Grammatiken, Wörterbücher, Editionen. Aber nicht bloß an materielle Instrumente wurde angeknüpft, auch an Gegenstände und

\footnotetext{
35 Kugler, Hartmut: »Zur literarischen Geographie des fernen Ostens im Parzival und im Jüngeren Titurel«. In: Wolfgang Dinkelacker/Ludger Grenzmann/Werner Höver (Hg.): Ja muz ich sunder riuwe sin (FS Karl Stackmann). Göttingen 1990, S. 107-147.
} 
Fragestellungen. Um nur Schlagwörter zu nennen: Das Werk Wolframs von Eschenbach ist ein zentraler Gegenstand der mediävistischen Forschung; Anpassungen an Phonemsysteme gehört zu den Analyseinstrumenten der Sprachkontaktforschung; Fragen der Raumentfaltung haben sich in den letzten Jahren zu einem Kernaspekt literaturwissenschaftlicher Forschung entwickelt. Das heißt, dass u.a. Arbeitsmittel, Gegenstände und Fragestellungen aus verschiedenen Teilfächern kombiniert wurden. Sie erscheinen damit nicht mehr als >Hilfswissenschaft<, sondern bieten Einsichten und Ansätze, an denen fruchtbar teilgenommen wird.

Welche Elemente für eine solche Kombination in Frage kommen, ist nicht beliebig, sondern wird von den Fächern ausgehandelt. Die Wissenschaft ist immer wieder gefordert, ihre Wissensbestände und Praktiken so zu hierarchisieren, dass sie auf verschiedenen Ebenen und auch für Fachfremde verfügbar bleiben und abgestuft zugänglich sind. Man muss beispielsweise keine vertieften Kenntnisse des Altfranzösischen aufweisen, um ein altfranzösisches Wörterbuch zu verwenden. Die Zusammenarbeit zwischen Experten setzt voraus, dass sie sich mit kleinem Zeitaufwand in einem fremden Gebiet rasch zurechtfinden können - und auch ihre Grenzen darin erkennen. Die Kombination verschiedener Ansätze ist nur dann fruchtbar, wenn man sich auf solche Hierarchien verständigen kann, andernfalls wird sie beliebig, denn es entfällt die Unterscheidung zwischen wichtig und unwichtig, die Wahl der Gegenstände ist dann vollends den institutionellen Machtstrukturen überlassen.

Die Ortsnamen sind ein Beispiel für die Schnittstelle zwischen Linguistik und Literaturwissenschaft, wenn auch ein abgelegenes. Dass sich das Gros der Fachkollegen dafür interessiert, ist nicht zu erwarten, es wäre auch nicht erstrebenswert. So stellt sich die Frage der Verallgemeinerbarkeit. Wie jedes Beispiel bringt es als Beispiel Probleme mit sich, denn Beispiele lenken vom Wesentlichen ab. Zwar geben sich Beispiele als verallgemeinerbare Spezialfälle, doch sie müssen Prämissen annehmen, die nicht allenthalben auf Zustimmung stoßen werden. Die Ausgangsfrage nach den Schnittmengen von Literaturwissenschaft und Linguistik geht von der weltfremden Annahme aus, eine Verständigung darüber sei möglich, was Literaturwissenschaft und was Linguistik eigentlich seien. Wenn man nach der Zusammenarbeit von der Linguistik und der Literaturwissenschaft fragt, suggeriert die Frage eine Einheitlichkeit, die empirisch nicht gegeben ist. Selbst innerhalb der Teilfächer hat eine erhebliche Differenzierung, um nicht zu sagen: Entfremdung, stattgefunden mit der Folge, dass man sich gegenseitig wie fachfremd erscheint. Zwar hat es Spezialisierungen, Auseinandersetzungen und Anfeindungen seit den ersten Tagen der Germanistik gegeben, doch die Entfernung voneinander erklärt sich nicht bloß durch ein vertieftes Expertentum, durch eine unübersichtliche Pluralität oder auch nur durch eine simple Machtfrage, sondern sie entsteht daraus, dass die Art und Weise des Zugangs des anderen gar nicht mehr nachvollzogen, sondern höchstens toleriert wird. So sei die Frage, die diesen Band prägt, variiert: Haben sich Germanistinnen und Germanisten zu Beginn des 21. Jahrhunderts (noch) etwas zu sagen?

Die Frage ist rhetorisch gestellt, doch sie hat ein beunruhigendes Potential. Die Germanistik hat heute mit den Herausforderungen neuer Veröffentlichungsmöglichkeiten und, im Feld dieser Möglichkeiten, auch mit neuen Konkurrenten umzugehen. Sie reagierte (leider) in den letzten Jahren mit einer allenthalben beklagten Publi- 
kationsflut, die nicht dazu geeignet ist, das Fach zu einen. Das Antidot gegen die Distanz unter Kollegen kann gleichwohl nicht darin bestehen, die Vielfalt des Faches oder seine Aktivitäten zu schmälern. Wünschenswert wäre dagegen ein verstärktes Bemühen darum, Erkenntnisinteresse und Ergebnisse so zu formulieren, dass sie innerhalb des Faches breit wahrgenommen werden können. Eine einfache Lösung für das Problem der Wahlverwandtschaften würde dann darin bestehen, sich gegenseitig Interesse für die Arbeiten des anderen zu unterstellen. Dann wäre man selber gefordert, alles dafür zu tun, dass künftige Leser aller Fachrichtungen in die Lage versetzt würden, die Arbeiten zu verstehen, mit ihnen umzugehen und im günstigsten Fall ihnen zu widersprechen.

Funding Open Access funding enabled and organized by Projekt DEAL.

Open Access Dieser Artikel wird unter der Creative Commons Namensnennung 4.0 International Lizenz veröffentlicht, welche die Nutzung, Vervielfältigung, Bearbeitung, Verbreitung und Wiedergabe in jeglichem Medium und Format erlaubt, sofern Sie den/die ursprünglichen Autor(en) und die Quelle ordnungsgemäß nennen, einen Link zur Creative Commons Lizenz beifügen und angeben, ob Änderungen vorgenommen wurden.

Die in diesem Artikel enthaltenen Bilder und sonstiges Drittmaterial unterliegen ebenfalls der genannten Creative Commons Lizenz, sofern sich aus der Abbildungslegende nichts anderes ergibt. Sofern das betreffende Material nicht unter der genannten Creative Commons Lizenz steht und die betreffende Handlung nicht nach gesetzlichen Vorschriften erlaubt ist, ist für die oben aufgeführten Weiterverwendungen des Materials die Einwilligung des jeweiligen Rechteinhabers einzuholen.

Weitere Details zur Lizenz entnehmen Sie bitte der Lizenzinformation auf http://creativecommons.org/ licenses/by/4.0/deed.de. 